\title{
Employment of Exosomes for Liquid Biopsies
}

\author{
Received: December 04, 2015; Accepted: December 10, 2015; Published: December \\ 17, 2015
}

Recently, the interest of research has been attracted by a new form of cell-to-cell communication mediated by cell-secreted vesicles known as exosomes. They range from 40 to $120 \mathrm{~nm}$ in size and are involved in normal and tumoral biological processes. Mainly, they mediate the intercellular communications both with the cells in the immediate vicinity of the secreting cells and with very distant cells by bloodstream [1]. This cell-to-cell communication is made possible by the transfer into recipient cells of the elements contained in (proteins, DNA and RNAs) and composing (trans-membrane and membrane anchored proteins, lipids) exosomes. These vesicles can be internalized by several routes, such as clathrin-dependent endocytosis, caveolinmediated uptake, macropinocytosis, phagocytosis, and lipid raftmediated internalization [2]. A growing number of studies have shown that these uptake mechanisms involve specific proteinprotein interactions, in particular among exosomal integrins, tetraspanins, proteoglycans and lectins with proteins, such as receptors, on target cell surface [2]. The pattern of external exosomal proteins constitute a code, still to be decrypted, that defines on which cell a vesicle will blend. However, it is still not known how much this uptake is specific, that is, whether it is at tissue or even at cell level. Maybe this pattern regulates also how wide or narrow the spectrum of exosome spread is $[3,4]$.

The microRNA content of the exosome differs from that of the parent cell, suggesting that some RNA molecules are selectively loaded into exosomes [5]. Remarkably, microRNAs sorted into exosomes can be regulated depending on the necessity of the parent cell [6]. This sorting mechanism seems due to specific sequence motifs lying in RNAs and recognized by proteins during exosomes biogenesis [7,8]. These export motifs constitute another biological language that resembles the nucleocytoplasmic RNA export language, motifs of which are collected in a recently published database [9]. Moreover, since the motifs could be bound by RNA-binding proteins, some tools such as SpliceAid [10] can be used to scan exosomal RNA sequences.

Owning to exosome uptake, the carried message can regulate gene expression of target cells and induce specific phenotypic changes, showing that exosome cargo molecules are functionally active [5]. These communication mechanisms are used also by tumor exosomes to modulate the tumor microenvironment, the invasion and migration of cancer cells, or even promoting the formation of the pre-metastatic niche [11,12]. Recently, it has also been discovered that cancer cells resistant to chemotherapy can transmit their resistance to other cancer cells via exosomes [13]. This mechanism vaguely resembles that of antibioticresistance transmitted by plasmids. A great deal of research effort

\section{Matteo Giulietti, Giulia Occhipinti, Alessandra Righetti, Tatiana Armeni, Giovanni Principato and Francesco Piva}

\author{
Department of Specialistic Clinical and \\ Odontostomatological Sciences, Polytechnic \\ University of Marche Region, Ancona, Italy
}

Corresponding author: Dr. Francesco Piva

f.piva@univpm.it

Department of Specialistic Clinical and Odontostomatological Sciences, Polytechnic University of Marche Region, Ancona, Italy.

Tel: +390712204683

Fax: +390712204609

Citation: Piva F. Employment of Exosomes for Liquid Biopsies. Transl Biomed. 2015, 6:4.

is addressed to identifying exosomal biomarkers that define who is the sender cell, the recipient cell and what is the meaning of the message. The role, as biomarkers, of some surface proteins or miRNAs has been recently elucidated [14,15]. For example, a recent work identified a membrane anchored protein that is specifically on exosome of pancreatic ductal adenocarcinoma so it can be used as bait to isolate tumour exosomes from blood of patients [16]. Obviously, there is still a great need to find the specific markers that distinguish exosomes of each kind of cancer and that, therefore, could be useful for non-invasive "liquid biopsies". In fact, exosomes are in all body fluids and from these fluids it will be possible to specifically isolate the tumor exosomes to be used as a means of disease diagnosis and therapeutic monitoring. Current research is focusing on the blockade of tumor exosome uptake, in order to prevent cancer cells from suggesting escape strategies to other cancer cells, increase their malignancy and avoid that a tumor controls the surrounding stroma or promotes the formation of a pre-metastatic niche in a distal tissue [2]. A further possibility is to remove tumor exosome directly from blood like in hemodialysis [17]. 
The "liquid biopsy" concept comprises also the analysis of free tumor DNA in the blood, but it has many limitations since circulating DNA molecules derive from death cells and it is not possible to know their exact tissue origin. However, it is very important to have genomic DNA originating from patient tumor in order to detect somatic mutations to be used for prognosis, stadiation, sub-type classification and therapeutic choice. The recent discovery that exosomes contain double-stranded DNA (dsDNA) of the parent cell $[18,19]$ along with the possibility to isolate exosomes released from a specific tissue or from a specific tumor via exosomal surface biomarkers will allow to obtain tumor DNA without performing a biopsy. The application of highly sensitive detection technologies, such as digital PCR, that virtually performs amplifications of each exosome, will allow the complete assessment of the tumor heterogeneity. This will also allow to realize the tumor heterogeneity, the selective effect of a therapy on a particular cancer cell population, the clonality and so the origin of a metastasis, the equilibrium among cancer cell populations.
However researchers have to be aware of the difficulties in working with exosomes, for example, dirty exosome isolations are not rare and are due to exosome tendency to bind plasma proteins. Isolations by isoelectric point do not precipitate all exosomes because of their different isoelectric points. Their stickiness to laboratory plastic can cause low isolation yields. Even the rotor angle of the centrifuge and the time from blood withdrawal to the analysis can influence the amount of collected exosomes. The sample has to be always treated to remove the microvesicles. Moreover, the recovery of tumor exosomes could be scarce since these exosomes are rare in clinical samples in comparison with those coming from normal cells, that outnumber tumor cells by several orders of magnitude. Therefore, many technology advancements and scientific discoveries are still needed to take full advantage of the potential of exosomes in clinical translation.

\section{Acknowledgments: None \\ Funding: None \\ Competing interests: None}




\section{References}

Camussi G, Deregibus MC, Bruno S, Cantaluppi V, Biancone L (2010) Exosomes/microvesicles as a mechanism of cell-to-cell communication. Kidney Int 78: 838-848.

Mulcahy LA, Pink RC, Carter DR (2014) Routes and mechanisms of extracellular vesicle uptake. J Extracell Vesicles 3.

Hoshino A, Costa SB, Shen TL, Rodrigues G, Hashimoto A, et al. (2015) Tumour exosome integrins determine organotropic metastasis. Nature 527: 329-335.

Rana S, Yue S, Stadel D, Zoller M (2012) Toward tailored exosomes: the exosomal tetraspanin web contributes to target cell selection. Int J Biochem Cell Biol 44: 1574-1584.

Zhang J, Li S, Li L, Li M, Guo C, et al. (2015) Exosome and exosomal microRNA: trafficking, sorting, and function. Genomics Proteomics Bioinformatics 13: 17-24.

6 Ragusa M, Statello L, Maugeri M, Barbagallo C, Passanisi R, et al. (2014) Highly skewed distribution of miRNAs and proteins between colorectal cancer cells and their exosomes following Cetuximab treatment: biomolecular, genetic and translational implications. Oncoscience 1: 132-157.

Batagov AO, Kuznetsov VA, Kurochkin IV (2011) Identification of nucleotide patterns enriched in secreted RNAs as putative cis-acting elements targeting them to exosome nano-vesicles. BMC Genomics 12 Suppl 3: S18.

Bolukbasi MF, Mizrak A, Ozdener GB, Madlener S, Strobel T, et al. (2012) miR-1289 and "Zipcode"-like Sequence Enrich mRNAs in Microvesicles. Mol Ther Nucleic Acids 1: e10.

Giulietti M, Milantoni SA, Armeni T, Principato G, Piva F (2015) ExportAid: database of RNA elements regulating nuclear RNA export in mammals. Bioinformatics 31: 246-251.
Piva F, Giulietti M, Burini AB, Principato G (2012) SpliceAid 2: a database of human splicing factors expression data and RNA target motifs. Hum Mutat 33: 81-85.

11 Zhang Y, Wang XF (2015) A niche role for cancer exosomes in metastasis. Nat Cell Biol 17: 709-711.

12 Santoni M, Piva F, Scarpelli M, Cheng L, Lopez BA, et al. (2015) The origin of prostate metastases: emerging insights. Cancer Metastasis Rev 34: 765-773.

13 Munoz JL, Bliss SA, Greco SJ, Ramkissoon SH, Ligon KL, et al. (2013) Delivery of Functional Anti-miR-9 by Mesenchymal Stem Cellderived Exosomes to Glioblastoma Multiforme Cells Conferred Chemosensitivity. Mol Ther Nucleic Acids 2: e126.

14. Mizoguchi M, Guan Y, Yoshimoto K, Hata N, Amano T, et al. (2013) Clinical implications of microRNAs in human glioblastoma. Front Oncol 3: 19.

15 Jayaram S, Gupta MK, Polisetty RV, Cho WC, Sirdeshmukh R (2014) Towards developing biomarkers for glioblastoma multiforme: a proteomics view. Expert Rev Proteomics 11: 621-639.

16 Melo SA, Luecke LB, Kahlert C, Fernandez AF, Gammon ST, et al. (2015) Glypican-1 identifies cancer exosomes and detects early pancreatic cancer. Nature 523: 177-182.

7 Marleau AM, Chen CS, Joyce JA, Tullis RH (2012) Exosome removal as a therapeutic adjuvant in cancer. J Transl Med 10: 134.

Kahlert C, MeloSA, Protopopov A, Tang J, SethS, et al. (2014) Identification of double-stranded genomic DNA spanning all chromosomes with mutated KRAS and p53 DNA in the serum exosomes of patients with pancreatic cancer. J Biol Chem 289: 3869-3875.

9 Thakur BK, Zhang H, Becker A, Matei I, Huang Y, et al. (2014) Doublestranded DNA in exosomes: a novel biomarker in cancer detection. Cell Res 24: 766-769. 